\title{
Hele-Shaw flow driven by an electric field
}

\author{
A. H. Khalid, N. R. McDonald and J. M. Vanden-Broeck \\ Department of Mathematics \\ University College London \\ Gower Street, London, WC1E 6BT, UK \\ Published on October 10, 2013
}

\begin{abstract}
The behaviour of two-dimensional finite blobs of conducting viscous fluid in a HeleShaw cell subject to an electric field is considered. The time-dependent free boundary problem is studied both analytically using the Schwarz function of the free boundary and numerically using a boundary integral method.

Various problems are considered including: (i) the behaviour of an initially circular blob of conducting fluid subject to an electric point charge located arbitrarily within the blob, (ii) the delay in cusp formation on the free boundary in sink driven flow due to a strategically placed electric charge and (iii) stability of exact steady solutions having both hydrodynamic and electric forcing.
\end{abstract}

Key words: Hele-Shaw flows, free boundary problems, complex variable methods.

\section{Introduction}

Hele-Shaw flows involve two-dimensional flow of a viscous Newtonian fluid in a thin gap between two parallel plates, a set-up first considered in experiments by Hele-Shaw in the late $19^{\text {th }}$ century [7]. Here, it is assumed the fluid is surrounded by a second fluid of negligible viscosity, such as air, the two fluids are immiscible and there is no surface tension. Hele-Shaw flows are mathematically analogous to those of groundwater flows, where the governing equations satisfy Darcy's law and have practical applications, for example, oil extraction [10, 16]. While straightforward to formulate as a mathematical problem, the free boundary evolution problem is nonlinear and is, in general, difficult to solve. For example, it is noteworthy that solutions describing fluid blobs with shrinking area (driven by a hydrodynamic sink) frequently breakdown in finite time owing to cusp formation or self-intersection of the fluid-air interface $[8,9]$.

The results presented may find applications in problems involving fluid extraction and in the theory of fluid flows in microfluidic devices, where the main interest lies in the manipulation of fluid blobs via electric fields. Entov and Etingof [4] raise the idea of Hele-Shaw free boundary flows and its application in electrokinetics, in which flow is induced by applying 
electric fields, known as 'electro-osmotic flow'. The introduction of applications of electroosmotic flow in microfluidic devices is also mentioned, where flow in narrow gaps or channels are driven by electric potential fields in $[11,22]$. More recently, controlling fluid blobs or bubbles in advanced microfluidic devices has become an area of interest for the purpose of bio-chemical processes and also in fluid logical devices $[2,13,17]$. A method known as electrowetting has become a widely used tool through a surge in so called 'lab-on-chip' devices for applications such as DNA analysis and medical diagnostics, where electrowetting, first introduced by Gabriel Lippmann in 1875 (English translation of paper in [15]), allows the manipulation of small drops or blobs of fluid on small surfaces, usually surrounded by air.

\subsection{Mathematical formulation and the Schwarz function approach}

In a Hele-Shaw cell let viscous fluid occupy the two-dimensional finite domain $\Omega(t)$ (the fluid 'blob') with a fluid-air interface denoted by $\partial \Omega(t)$ (the free boundary). The resulting motion of $\partial \Omega(t)$ is determined by the pressure gradient acting at the interface, resulting from hydrodynamic forces and any external body forces acting on the fluid, e.g. gravity. Determining the evolution of $\Omega(t)$ is widely known as the Hele-Shaw free boundary problem [6, 16]. The pressure in the blob satisfies Laplace's equation and often such problems are referred to as Laplacian growth problems, where the flow is driven by hydrodynamic singularities located within $\Omega(t)$, and the normal velocity of $\partial \Omega(t)$ is given by the normal gradient of a function satisfying Laplace's equation. The velocity field averaged over the thin gap between the two plates is given by

$$
\tilde{\boldsymbol{u}}=-\frac{h^{2}}{12 \mu} \nabla \tilde{p}+\rho \tilde{\boldsymbol{g}}
$$

where $\tilde{p}$ is the pressure, the coefficient $h^{2} / 12 \mu$ represents the mobility of the fluid ( $h$ is the gap width between the two plates, $\mu$ is the viscosity of the fluid), $\rho$ is the density of the fluid and $\tilde{\boldsymbol{g}}$ represents a body force due to some external field [4]. Here the body force is assumed to be conservative, i.e.

$$
\tilde{\boldsymbol{g}}=\nabla \tilde{\Psi}
$$

where $\tilde{\Psi}(x, y)$ is a scalar potential. Since the fluid is incompressible, conservation of mass gives

$$
\nabla \cdot \tilde{\boldsymbol{u}}=\sum_{j=1}^{N} \tilde{Q}_{j} \delta\left(x-x_{j}, y-y_{j}\right),
$$

where $\tilde{Q}_{j}$ are the strengths of hydrodynamic point sources or sinks within $\Omega(t)$ located at positions $\boldsymbol{x}_{\boldsymbol{j}}=\left(x_{j}, y_{j}\right)$. The right hand side of (3) can be appropriately generalised to include higher order hydrodynamic singularities e.g. dipoles, quadrupoles etc. It has been shown in [4], after non-dimensionalisation (dropping the tildes), the mathematical formulation of the 
problem can be written as the following free boundary problem:

$$
\begin{array}{rlrl}
\nabla^{2} \phi & =\sum_{j=1}^{N} Q_{j} \delta\left(x-x_{j}, y-y_{j}\right), & & (x, y) \in \Omega(t), \\
\phi & =\Psi(x, y), & (x, y) \in \partial \Omega(t), \\
v_{n} & =\frac{\partial \phi}{\partial n}, & (x, y) \in \partial \Omega(t),
\end{array}
$$

where the (real) velocity potential satisfies $\phi(x, y)=-(p-\Psi)$ and $v_{n}$ is the normal velocity of the free boundary.

The case of $\Psi \equiv 0$ is the classical Hele-Shaw free boundary problem, see e.g. [3, 5, 19]. Richardson introduced harmonic moments and showed that they were all conserved during the flow evolution except the zeroth order moment which, being equivalent to blob area, changed according to the net mass injected by the hydrodynamic singularities [18]. The moment description provides a method with which the shape of the evolving free boundary can be determined, where the problem is reduced to the solution of a system of algebraic equations.

An alternative approach to finding exact solutions employs the Schwarz function of the free boundary. In the complex plane $z=x+i y$, the Schwarz function of the free boundary is defined as $g(z, t)=\bar{z}$ on $z \in \partial \Omega(t)$, where $g$ is an analytic function in the neighbourhood of $\partial \Omega(t)$. It has been shown that

$$
\frac{\partial w}{\partial z}=\frac{1}{2} \frac{\partial g}{\partial t}+\frac{\partial M}{\partial z}
$$

holds on the free boundary $\partial \Omega(t)$, where

$$
M(z)=\Psi((z+g(z)) / 2,(z-g(z)) /(2 i))
$$

is an analytic function in the neighbourhood of $\partial \Omega$ and $w(z, t)$ is the complex potential of the flow [14]. Since both sides of (5) are analytic in the neighbourhood of $\partial \Omega(t)$, analytic continuation allows for (5) to be valid over the entire fluid domain $\Omega(t)$. In the absence of any background fields $(M \equiv 0)$, (5) reduces to the well-known Schwarz function equation governing the flow $[3,14]$.

Some non-trivial steady exact solutions of the problem given by (4) have previously been found $[4,14]$. Such solutions can be derived, as shown in [14], by considering the balance between the terms $\partial w / \partial z$ and $\partial M / \partial z$ of (5) as singularities inside $\Omega(t)$ are approached. The stability of the steady solutions found in $[4,14]$ is an open question and is investigated numerically in $\S 6$.

\subsection{Flows driven by external fields}

When hydrodynamic singularities within the fluid domain $\Omega(t)$ are absent, the free boundary evolves only under the action of the external field $\Psi$ and satisfies the following free boundary 
problem

$$
\begin{aligned}
& \nabla^{2} \phi=0, \quad(x, y) \in \Omega(t), \\
& \phi=\Psi(x, y), \quad(x, y) \in \partial \Omega(t), \\
& v_{n}=\frac{\partial \phi}{\partial n}, \quad(x, y) \in \partial \Omega(t) \text {. }
\end{aligned}
$$

The task is to find $\partial \Omega(t)$ of a fluid blob evolving under the influence of a background potential field given the initial boundary $\partial \Omega(0)$. Since there are no hydrodynamic singularities driving the flow, the derivative of the complex potential $\partial w / \partial z\left(=w^{\prime}(z, t)\right)$ has no singularities in the fluid domain $\Omega(t)$ and hence the left hand side of (5) is regular. The unknown boundary $\partial \Omega(t)$ is parameterised by a conformal map $z=f(\zeta, t)$ from the interior of the unit $\zeta$-disk to the interior of the fluid blob $\Omega(t)$. In general, the map $f(\zeta, t)$ is unknown but can often be "guessed" or deduced up to unknown time-varying parameters. By balancing singular terms of (5), the aim is to deduce ordinary differential equations for the unknown time-dependent parameters of the conformal map $z=f(\zeta, t)$, and subsequently determine the evolution of the free boundary $\partial \Omega(t)$. This approach is used to investigate the stability and behaviour of initially circular blobs of fluid for the case when the external field is an electric point charge.

\section{Analytical results}

\subsection{Steady solution for an initially circular fluid blob with a point charge at its centre}

Consider a circular blob of conducting viscous fluid with radius $R$ centred at the origin enclosing a point charge located at $z=0$, i.e. within the initial fluid domain $\Omega(0)$. By symmetry it is expected that this be an exact steady solution. The external potential owing to the point charge of strength $E$ centred at $z=0$ is given by $\Psi=(E / 2 \pi) \log r=M(z)$ on $\partial \Omega(t)$, where

$$
M=\frac{E}{4 \pi} \log (z g) .
$$

Employing (8), the Schwarz function equation (5) becomes

$$
\frac{\partial w}{\partial z}=\frac{1}{2} \frac{\partial g}{\partial t}+\frac{E}{4 \pi}\left(\frac{1}{z}+\frac{g_{z}}{g}\right)
$$

The boundary of a circular blob of radius $R(t)$ centred at $z=0$ has Schwarz function $g(z, t)=R^{2} / z$. Using (9) and considering the singular structure of terms in (9) as $z \rightarrow 0$ and recalling there is no singularity in $w$ since there are no hydrodynamic sources, it follows that $\dot{R}=0$. This gives, as expected, a steady solution in which the fluid blob remains circular with constant radius $R_{0}=R(0)$, irrespective of the sign of $E$. 


\subsection{Stability of the free boundary subject to an electric point charge}

The stability of the circular blob equilibria is now studied with $R_{0}=1$, where a small perturbation to the circular boundary is imposed. Consider the conformal map from the unit $\zeta$-disk to a nearly-circular fluid blob with a small disturbance to the initial boundary, $\partial \Omega(0)$, given by (see e.g. $[3,5,6]$ )

$$
z=\zeta+\alpha \zeta^{n},
$$

where $n$ is an integer such that $n \geq 2$ and $\alpha(t)$ is a real time varying coefficient such that $|\alpha| \ll 1$. In polar coordinates with $r=|z|$ (10) is equivalent to $r=1+\alpha \cos (n-1) \theta+\mathcal{O}\left(\alpha^{2}\right)$, $0 \leq \theta<2 \pi$. Using $\bar{\zeta}=\zeta^{-1}$ and inversion of (10), the Schwarz function behaves like

$$
g(z, t) \rightarrow \frac{\alpha}{z^{n}}+\frac{n \alpha^{2}+1}{z}+\mathcal{O}\left(z^{n-2}\right), \quad \text { as } z \rightarrow 0 .
$$

Ignoring terms of $\mathcal{O}\left(\alpha^{2}\right)$ and considering the structure of the singularities of $\mathcal{O}\left(z^{-n}\right)$ in (9), the following ordinary differential equation for $\alpha(t)$ is obtained:

$$
\dot{\alpha}=\frac{E}{2 \pi}(n-1) \alpha,
$$

which has exponentially decaying solution for a negative point charge $E$. For $E>0$, the amplitude of the disturbances grow exponentially with time, and the assumption of small $\alpha$ breaks down. Hence, the flow is stable only if the point charge within the fluid domain is negative. Physically, this stability property owes itself to the following property: anomalous fluid outside $|z|=1$ is attracted inward by a negative point charge, while a fluid deficit inside $|z|=1$ is pushed outward. Together, these processes re-symmetrise a perturbed circular blob. For a positive point charge an anomaly for $|z|=1$ is pushed further outward leading to instability. This stability prediction is tested numerically in $\S 4.1$ (see Figure 1).

It is worth noting that exact time-dependent solutions which combine both hydrodynamic singularities and point charges have not yet been found. A prime candidate for generating such solutions is to use maps of the type (10) since these yield explicit solution in the case driven by hyrodynamic singularities when point charges are absent (e.g. see [3, 6]). However when combined with point charges this map does not yield solution with, for example, the correct rate of change of area. Finding a class of maps which admits such exact solutions is an open question.

\subsection{Circular fluid blob with an off-centre point charge}

Suppose the circular blob in $\S 2.1$ is off-set by a small amount $\epsilon$ so that it is now centred at $z=\epsilon$, with the point charge $E$ located at $z=0$ which lies within the fluid domain $\Omega(t)$. Without loss of generality $\epsilon$ is assumed to be real. Making the approximation that, to leading order, the blob remains circular throughout its evolution, consider the conformal map from the unit $\zeta$-disk to the fluid blob given by

$$
z(\zeta, t)=\epsilon+R \zeta,
$$


where $\epsilon(t)$ and $R(t)$ are real time varying coefficients to be found. The validity of the approximation that the blob remains circular during its evolution is tested by comparing the analytical results of this section with numerical results in $§ 4.1$. The map (13) implies $\partial \Omega(t)$ has the Schwarz function

$$
g(z, t)=\epsilon+\frac{R^{2}}{z-\epsilon}
$$

Expanding (14) for small $\epsilon$

$$
g(z, t) \rightarrow \epsilon+\frac{R^{2}}{z}+\frac{R^{2} \epsilon}{z^{2}}+\mathcal{O}\left(\epsilon^{2}\right)
$$

Considering the structure of the singularities of $\mathcal{O}\left(z^{-1}\right)$ and $\mathcal{O}\left(z^{-2}\right)$ on both sides of (9), the following pair of ordinary differential equations are obtained for $R(t)$ and $\epsilon(t)$ :

$$
\begin{aligned}
R \dot{R} & =0, \\
\frac{R^{2} \dot{\epsilon}}{2}+\epsilon R \dot{R}-\frac{E}{4 \pi} \epsilon & =0 .
\end{aligned}
$$

Hence, (16a) gives $R(t)$ is constant, denoted by $R_{0}$, the radius of the initial fluid blob, which is consistent with conservation of mass. Therefore, (16b) gives

$$
\epsilon(t)=\epsilon_{0} \exp \left(\frac{E}{2 R_{0}^{2} \pi} t\right),
$$

where $\epsilon_{0}$ denotes the abscissa of the centre of the initial circular fluid blob. Again, it is clear for a negative point charge, $E<0$, the resulting flow is stable and the blob moves through a series near-circular domains $\Omega(t)$, until a steady state is achieved where $\epsilon(t) \rightarrow 0$. That is, the blob approaches a state where it is circular and centre coinciding with the location of the point charge. In the case for a positive charge, $E>0$, the flow is unstable, since $\epsilon(t)$ grows exponentially and the asymptotic analysis breaks down and it is unlikely the blob remains near-circular. These predictions are tested numerically in $\S 4.1$.

\section{Numerical method}

\subsection{Mathematical formulation}

Consider the complex velocity potential $w^{\prime}(z)=u-i v=\partial \phi / \partial x-i \partial \phi / \partial y$, which is an analytic function of $z=x+i y$ except at hydrodynamic singularities, such as sources and sinks. In the numerical procedure the fluid velocity $(u, v)$ is to be found on the boundary $\partial \Omega(t)$, which is then used to advect the boundary. The Cauchy integral formula for some $z_{m}$ on the boundary $\partial \Omega(t)$ (with positive orientation) gives

$$
\frac{1}{2 \pi i} \oint_{\partial \Omega(t)} \frac{w^{\prime}(z)}{z-z_{m}} d z=\frac{1}{2} w^{\prime}\left(z_{m}\right)+\gamma,
$$

where $\gamma=\sum_{k}\left[\operatorname{Res}\left(w^{\prime}(z) / z-z_{m} ; z_{k}\right)\right]$ and $z_{k}, k=1, \ldots K$, represent $K$ hydrodynamic singularities of $w^{\prime}(z)$ lying within the fluid domain $\Omega(t)$ at locations $z=z_{k}$. The singular 
behaviour of $w^{\prime}(z)$ is known when the flow is driven by singularities such as sources or sinks. In the absence of hydrodynamic singularities $\gamma \equiv 0$.

Letting $w^{\prime}(z)=u-i v$, the left hand side of (18) is

$$
I=\oint_{\partial \Omega(t)} \frac{u-i v}{z-z_{m}} d z=\oint_{\partial \Omega(t)} \frac{\left(u \tilde{x}_{m}-v \tilde{y}_{m}\right)-i\left(v \tilde{x}_{m}+u \tilde{y}_{m}\right)}{\tilde{x}_{m}^{2}+\tilde{y}_{m}^{2}}(d x+i d y),
$$

where $\tilde{x}_{m}=\left(x-x_{m}\right)$ and $\tilde{y}_{m}=\left(y-y_{m}\right)$. Considering the imaginary part of (18) gives

$$
\oint_{\partial \Omega(t)}\left[\frac{-\left(v \tilde{x}_{m}+u \tilde{y}_{m}\right) d x+\left(u \tilde{x}_{m}-v \tilde{y}_{m}\right) d y}{\tilde{x}_{m}^{2}+\tilde{y}_{m}^{2}}\right]=\pi u\left(z_{m}\right)+\Im\{2 \pi i \gamma\} .
$$

Now, parameterising the free boundary $\partial \Omega(t)$ with the arc length $s$, such that $x=x(s, t)$ and $y=y(s, t)$, it follows

$$
\left(\frac{\partial x}{\partial s}\right)^{2}+\left(\frac{\partial y}{\partial s}\right)^{2}=1
$$

where $0 \leq s<L(t)$ and $L(t)$ denotes the total length of $\partial \Omega(t)$ at time $t$. Note, the integral $I$ can be written as a Cauchy Principal Value integral:

$$
I=\mathrm{PV} \int_{0}^{L(t)} \frac{u(s)-i v(s)}{z(s)-z\left(s_{m}\right)} \frac{d z}{d s} d s
$$

where $z_{m}=z\left(s_{m}\right)$. Considering (20), on which $u$ and $v$ are also functions of $s$, then

$$
\mathrm{PV} \int_{0}^{L(t)}\left[\frac{-\left(v \tilde{x}_{m}+u \tilde{y}_{m}\right) \frac{d x}{d s}+\left(u \tilde{x}_{m}-v \tilde{y}_{m}\right) \frac{d y}{d s}}{\tilde{x}_{m}^{2}+\tilde{y}_{m}^{2}}\right] d s=\pi u\left(z_{m}\right)+\Im\{2 \pi i \gamma\},
$$

where $d x / d s$ and $d y / d s$ denote the derivatives of $x$ and $y$ with respect to $s$. Hence, given $\partial \Omega(t),(23)$ is an integral equation for unknown velocity field $(u, v)$ at time $t$.

Since the boundary $\partial \Omega(t)$ is an equipotential surface given by $\Phi(x, y, t)=\phi(x, y, t)-$ $\Psi(x, y)=0$, the derivative of $\Phi$ with respect to arc length along $\partial \Omega(t)$ gives

$$
\frac{\partial \Phi}{\partial s}=\frac{\partial \Phi}{\partial x} \frac{\partial x}{\partial s}+\frac{\partial \Phi}{\partial y} \frac{\partial y}{\partial s}=\left(u-\frac{\partial \Psi}{\partial x}\right) \frac{\partial x}{\partial s}+\left(v-\frac{\partial \Psi}{\partial y}\right) \frac{\partial y}{\partial s}=0
$$

which is equivalent to the dynamic boundary condition (4b). Solution to (23) and (24) provide the velocity $(u, v)$ on the boundary for given $\partial \Omega(t)$.

Once $u$ and $v$ are found on $\partial \Omega(t)$, the evolution of the free boundary is found by using

$$
\begin{aligned}
& \frac{d x}{d t}=u, \\
& \frac{d y}{d t}=v
\end{aligned}
$$

which is equivalent to the kinematic boundary condition (4c). 


\subsection{Numerical procedure}

At a particular time $t=t_{j}$ the numerical solution of the linear integro-differential system (21), (23), (24) and (25) is considered. The boundary $\partial \Omega\left(t_{j}\right)$ is parameterised with respect to arc length $s$ in the anti-clockwise direction, with total arc length $L\left(t_{j}\right)$. Equation (23) applies where $N+1$ equispaced mesh points are defined on the interval $\left[0, L\left(t_{j}\right)\right]$, such that

$$
\begin{aligned}
S_{1} & =0, \\
S_{i+1} & =S_{i}+\Delta S,
\end{aligned}
$$

for $i=, 1 \ldots, N$, where the equispaced mesh size is given by

$$
\Delta S=\frac{L\left(t_{j}\right)}{N}=\frac{S_{N+1}}{N},
$$

and $S_{N+1}=L\left(t_{j}\right)$.

New variables for the quantities of interest on $\partial \Omega\left(t_{j}\right)$ are introduced:

$$
\begin{aligned}
X_{i, j} & =x\left(S_{i}, t_{j}\right), \\
Y_{i, j} & =y\left(S_{i}, t_{j}\right), \\
U_{i, j} & =u\left(S_{i}, t_{j}\right), \\
V_{i, j} & =v\left(S_{i}, t_{j}\right),
\end{aligned}
$$

for $i=1, \ldots, N+1$. Since the boundary $\partial \Omega\left(t_{j}\right)$ is closed, this enforces the condition

$$
\begin{aligned}
X_{N+1, j} & =X_{1, j}, \\
Y_{N+1, j} & =Y_{1, j}, \\
U_{N+1, j} & =U_{1, j}, \\
V_{N+1, j} & =V_{1, j} .
\end{aligned}
$$

The boundary at the next time-step $t=t_{j+1}$ is found from (25), which in terms of variables in (28) gives

$$
\begin{aligned}
\hat{x}_{i, j+1} & =X_{i, j}+U_{i, j} \Delta t, \\
\hat{y}_{i, j+1} & =Y_{i, j}+V_{i, j} \Delta t .
\end{aligned}
$$

The points $\left(\hat{x}_{i, j+1}, \hat{y}_{i, j+1}\right), i=1, \ldots, N+1$, are then linearly interpolated to an equispaced mesh with respect to arc length of $\partial \Omega\left(t_{j+1}\right)$, as described above, to give $\left(X_{i, j+1}, Y_{i, j+1}\right)$. Accuracy of the time-stepping routine can be increased by using, for example, Runge-Kutta methods for marching forward in time.

The above procedure is reliant on determining the $2 N$ unknown quantities $\left(U_{i, j}, V_{i, j}\right)$, $i=1, \ldots, N$, by solving the system of equations (23) and (24), which translate, upon discretisation, to a linear system for the velocity field $(u, v)$ on the boundary. Together, (23) and (24) provide $2 N$ equations, where the points $\left(x_{m}, y_{m}\right)=\left(x\left(s_{m}, t_{j}\right), y\left(s_{m}, t_{j}\right)\right) \in \partial \Omega\left(t_{j}\right)$ lie at the midpoints of the equispaced mesh, i.e. $X_{i+\frac{1}{2}, j}=x\left(S_{i+\frac{1}{2}}, t_{j}\right), Y_{i+\frac{1}{2}, j}=y\left(S_{i+\frac{1}{2}}, t_{j}\right)$, where $S_{i+\frac{1}{2}}=\left(S_{i}+S_{i+1}\right) / 2$ for $i=1, \ldots, N$. 
Note, since the boundary is closed, there exist $N$ distinct midpoints, as well as mesh points, on the interval $\left[0, L\left(t_{j}\right)\right]$. Choosing the singular points $\left(x_{m}, y_{m}\right)$, i.e. $z_{m}=x_{m}+i y_{m}$, to be at the midpoints of the equispaced mesh invites use of the trapezoidal rule in order to calculate the integral in (23). The symmetry of the trapezoidal rule and the discretisation with respect to the singular points of the integrand allow for the evaluation of the integral by ignoring the singularities with an accuracy no less than that of a non-singular integral [21]. Hence, the integral in (23) is replaced by a sum and at time $t=t_{j}$ the equation can be discretised as

$$
\begin{array}{r}
\sum_{i=1}^{N}\left[\frac{-\left(V_{i, j} \tilde{x}_{l, j}+U_{i, j} \tilde{y}_{l, j}\right)\left(\frac{d x}{d s}\right)_{i, j}+\left(U_{i, j} \tilde{x}_{l, j}-V_{i, j} \tilde{y}_{l, j}\right)\left(\frac{d y}{d s}\right)_{i, j}}{\tilde{x}_{l, j}^{2}+\tilde{y}_{l, j}^{2}}\right] \Delta S= \\
=\pi U_{l+\frac{1}{2}, j}+\Im\left\{2 \pi i \Gamma_{l, j}\right\},
\end{array}
$$

for $l=1, \ldots, N$, where $\tilde{x}_{l, j}=X_{i, j}-X_{l+\frac{1}{2}, j}$ and $\tilde{y}_{l, j}=Y_{i, j}-Y_{l+\frac{1}{2}, j}$. The derivatives $(d x / d s)_{i, j}$ and $(d y / d s)_{i, j}$ are calculated via finite difference, and $\Gamma_{l, j}$ represents the contribution from the residues due to the $K$ hydrodynamic singularities lying within the contour $\partial \Omega\left(t_{j}\right)$ at the $l^{\text {th }}$ midpoint. The values $U_{l+\frac{1}{2}, j}$ are found by interpolating $U_{l, j}$ at neighbouring mesh points. Thus (31) represents $N$ equations $(l=1, \ldots, N)$ in $2 N$ unknowns $\left(U_{i, j}, V_{i, j}\right)$ for $i=1, \ldots, N$.

Finally, in discrete form, (24) at time $t=t_{j}$ is written as

$$
\left(U_{i, j}-\left(\frac{d \Psi}{d x}\right)_{i, j}\right)\left(\frac{d x}{d s}\right)_{i, j}+\left(V_{i, j}-\left(\frac{d \Psi}{d y}\right)_{i, j}\right)\left(\frac{d y}{d s}\right)_{i, j}=0
$$

where $(d \Psi / d x)_{i, j}$ and $(d \Psi / d y)_{i, j}$ are given on $\partial \Omega\left(t_{j}\right)$ at the mesh points for $i=1, \ldots, N$. This gives a further $N$ equations for $\left(U_{i, j}, V_{i, j}\right)$. Thus, the $2 N$ unknowns $\left(U_{i, j}, V_{i, j}\right), i=$ $1, \ldots, N$, are found by solving the set of $2 N$ linear equations given by (31) and (32) as a $2 N \times 2 N$ matrix system at time $t=t_{j}$. The linear (matrix) system is solved using Gaussian elimination via MATLAB's backslash command.

\section{Numerical results}

The numerical procedure is checked by comparing numerical results with the trivial solution of a growing circular blob with a source at the centre, and also for the case where the initial boundary is given by $z=\zeta+a \zeta^{2}, a \in \mathbb{R}$, driven by a source, where the boundary tends to a circle centred about the source location. This latter case having a well-known exact solution (e.g. see [3, 6]). Typical choices of discretisation for a blob with $\mathcal{O}(\pi)$ area is $\Delta s \sim \mathcal{O}\left(10^{-2}\right)$ and $\Delta t \sim \mathcal{O}\left(10^{-3}\right)$. The numerical results converge with good agreement to the exact solutions.

\subsection{Circular blob subject to an electric point charge}

The stability of a circular fluid blob subject to point charge, discussed in $\S 2.2$, is tested numerically. The boundary given by the map (10) is considered, with $\alpha=0.1, n=6$ and an electric point charge is located at the origin. Figure 1a demonstrates stability for a 


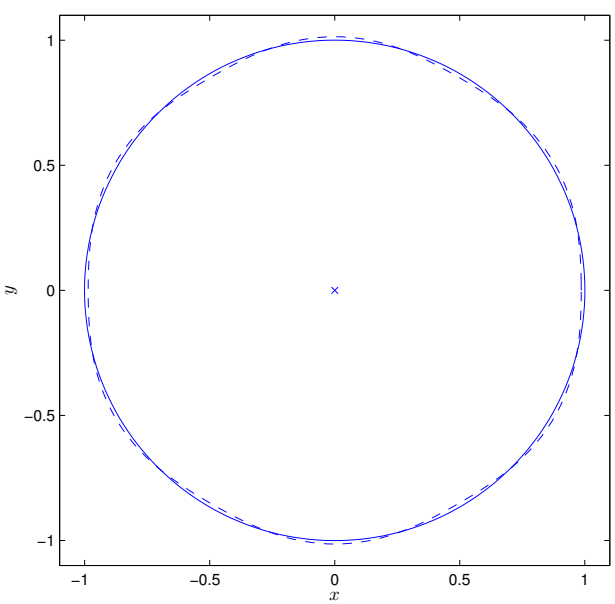

(a)

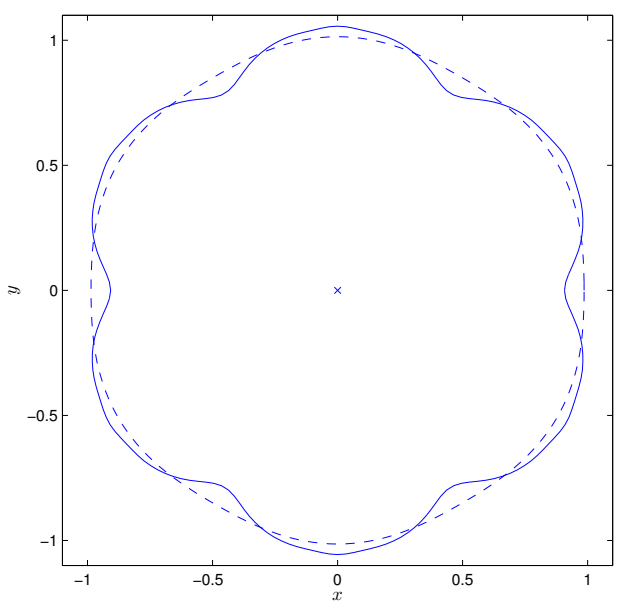

(b)

Figure 1: Stability of a perturbed circular blob for (a) $E=-\pi$ and (b) $E=\pi$. Initial boundary (dashed) given by (10) with $\alpha=0.1$ and $n=6$. Boundary shapes are shown at later times (solid) given at (a) $t=4$ and (b) $t=0.5$. Here there are $N=200$ mesh points on the boundary and step-size $\Delta t=1.25 \times 10^{-4}$

negative point charge, where disturbances on the boundary are suppressed and the boundary approaches a circle. Whereas in figure 1b, as predicted, for a positive point charge the instability is clear where the disturbances on the boundary grow and the solution rapidly breaks down. For this reason only the stable case of $E<0$ is investigated in the following numerical experiments.

The analytic solutions presented in $\S 2.3$ are also compared with numerical results. At time $t=0$, a circular blob with radius $R_{0}=1$ centred at $\left(\epsilon_{0}, 0\right)$ is allowed to evolve owing to a fixed electric point charge at the origin with strength $E<0$.

Here, the main interest is in the quantity $\epsilon(t)$ given in (17), i.e. the $x$-coordinate of the circular fluid blob. For comparison purpose, the centre of mass $\bar{\epsilon}$ of the numerically computed blob is defined

$$
\bar{\epsilon}(t)=\frac{1}{A} \iint_{\Omega(t)} x d x d y
$$

where $A$ is the area of the fluid blob and computed numerically. The analytic solution, $\epsilon(t)$, is compared with the numerical results for $\bar{\epsilon}(t)$.

The behaviour of $\bar{\epsilon}(t)$ with $t$ is shown in figure 2 for different values of electric point charge, $E$, with $N=125$ mesh points on the boundary and step-size $\Delta t=1 \times 10^{-4}$. Initially $\epsilon_{0}=0.1$ and $E=-\pi / 2,-\pi$ and $-2 \pi$. The numerical and exact solutions are superimposed in figure 2 and are in excellent agreement. Plots of $\partial \Omega(t)$ (not shown) establish that the boundary remains very close to circular throughout its motion.

The numerical method is used to investigate the evolution of the boundary for larger choices $\epsilon_{0}$ for which the analytical result does not necessarily hold (being a small- $\epsilon$ theory). Figure 3 shows the evolution of the centre of mass, $\bar{\epsilon}(t)$, compared with prediction from the analytic solution, $\epsilon(t)$, in the cases $\epsilon_{0}=0.25$ and $\epsilon_{0}=0.5$. It is apparent that there is no longer excellent agreement between the analytical and numerical solutions, as expected, 


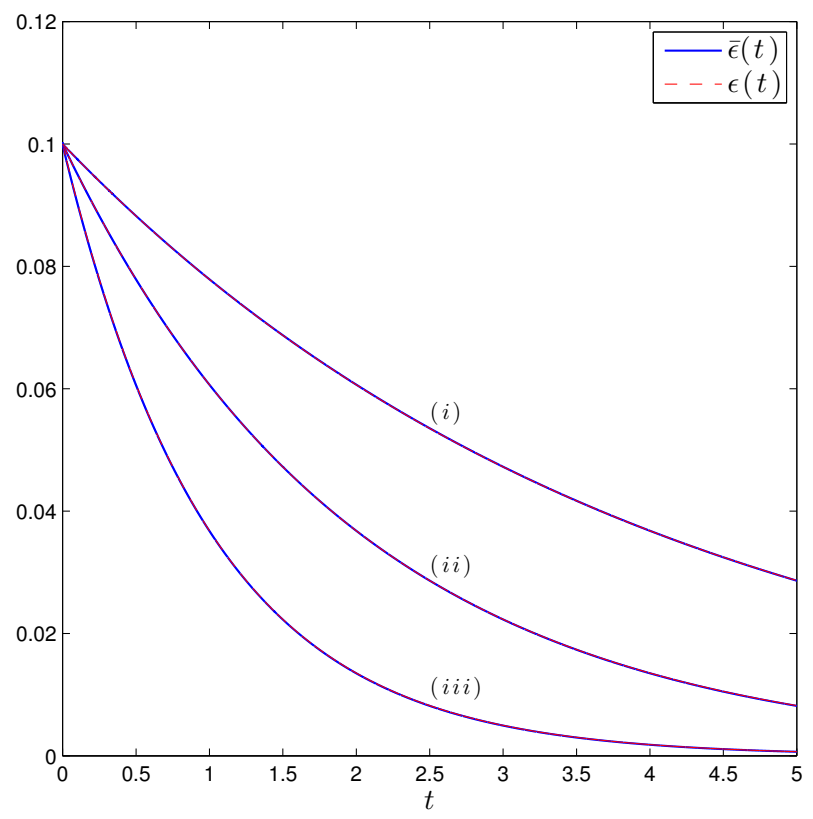

Figure 2: Evolution of the centre of mass, $\bar{\epsilon}(t)$, superimposed with the analytic solution (17) for the centre of the circle, $\epsilon(t)$, of a circular fluid blob with initial centre $\left(\epsilon_{0}, 0\right)$ such that $\epsilon_{0}=0.1$ and radius $R_{0}=1$. Three cases for increasing point charge strength are shown: (i) $E=-\pi / 2$, (ii) $E=-\pi$ and (iii) $E=-2 \pi$.

since the assumption of small $\epsilon$ is made in $\S 2.3$. Nevertheless, even in this non-asymptotic regime the comparison is good. Figures 4 and 5 show snap shots of the evolving boundary corresponding to the results of figure 3. It is clear the boundary shape remains closely circular during its evolution and by $t=10$ the blob is circular with centre coinciding with the point charge.

The case in which $\epsilon_{0}$ is chosen such that the electric point charge is close to the initial boundary (i.e. $\epsilon_{0}$ comparable to $R_{0}$ ), but still within the fluid domain, is also studied. For such a set up the evolution of the boundary is shown in figure 6. Initially, a 'pinching' of the boundary can be seen near the point charge (see figure 6b), after which the blob evolves through a smooth sequence of elliptical and egg-like domains. Finally, for large time the fluid blob tends to the same steady state as for small $\epsilon_{0}$. That is, the fluid blob becomes symmetrised about the location of the point charge, adopting a circle of radius $R_{0}$ centred at the origin.

A 'random' boundary shape, $\partial \Omega(0)$, of area $\pi$ is generated using [20]. The numerical procedure is used to compute the evolution of the blob with a negative point charge located within the closed boundary. The expected symmetrising of the fluid blob to a unit circle about the point charge is demonstrated in figure 7 . 


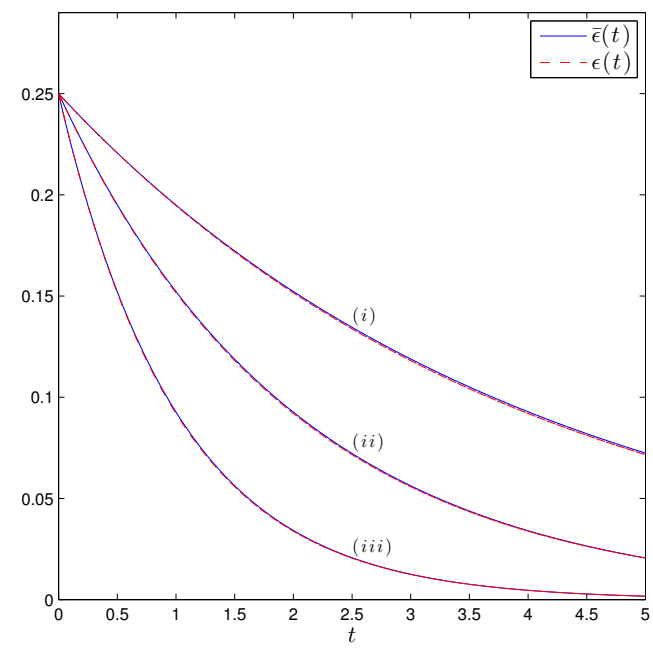

(a) $\epsilon_{0}=0.25$

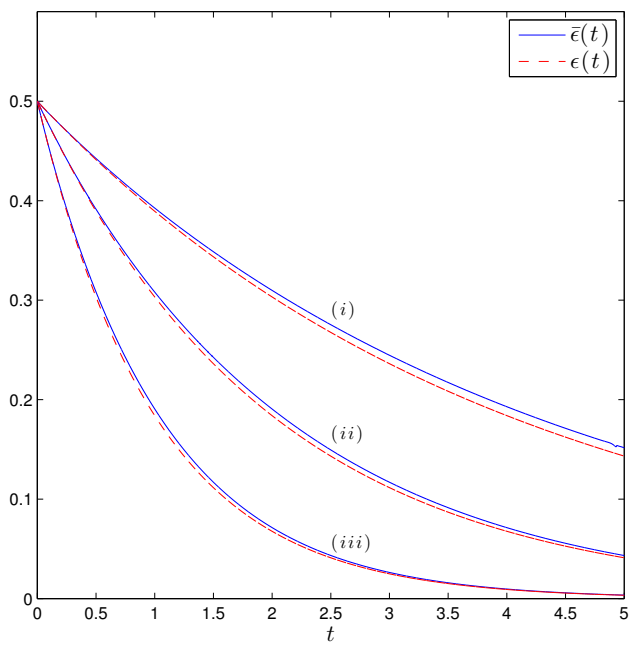

(b) $\epsilon_{0}=0.5$

Figure 3: Evolution of the centre of mass, $\bar{\epsilon}(t)$, superimposed with the analytic solution (17) for the centre of the circle, $\epsilon(t)$, of an initially circular fluid blob centred at $\left(\epsilon_{0}, 0\right)$ with radius $R_{0}=1$. Three cases for increasing point charge strength are shown: (i) $E=-\pi / 2$, (ii) $E=-\pi$ and (iii) $E=-2 \pi$.

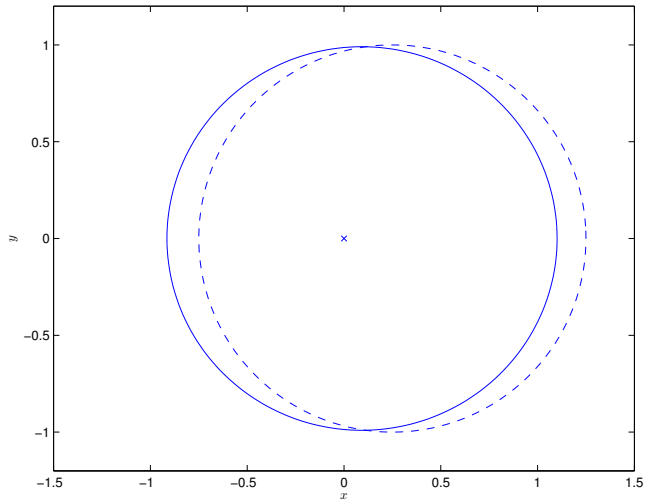

(a) $t=1$

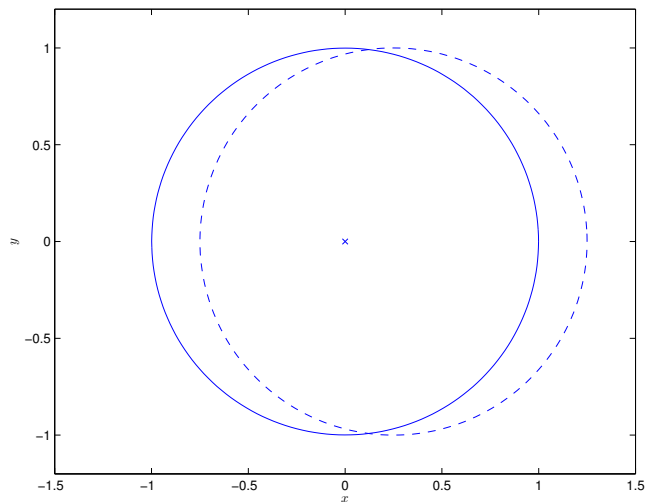

(b) $t=10$

Figure 4: Snapshots of evolving boundary (solid) of initially circular fluid blob (dashed) of radius $R_{0}=1$ centred at $\left(\epsilon_{0}, 0\right)$ with $\epsilon_{0}=0.25$. An electric point charge of strength $E=-\pi$ is located at the origin (marked by cross). 


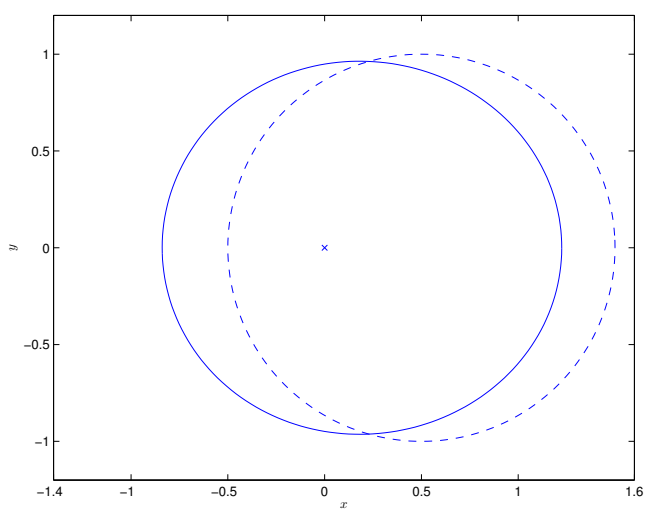

(a) $t=1$

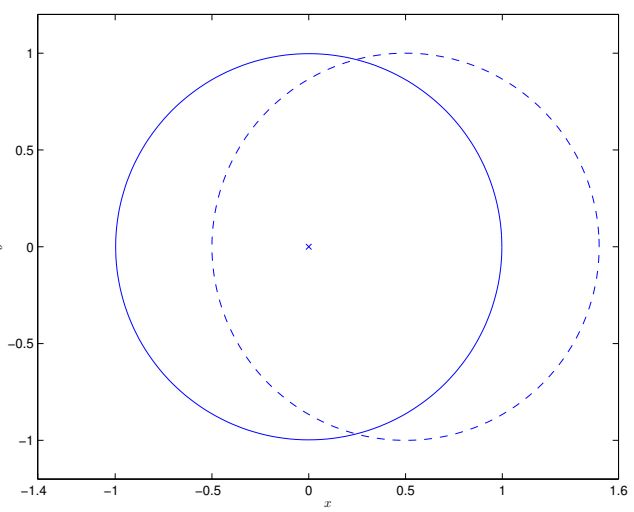

(b) $t=10$

Figure 5: Snapshots of evolving boundary (solid) of initially circular fluid blob (dashed) of radius $R_{0}=1$ centred centred at $\left(\epsilon_{0}, 0\right)$ with $\epsilon_{0}=0.5$. An electric point charge of strength $E=-\pi$ is located at the origin (marked by cross).

\section{Delaying cusp formation in sink driven flows using an electric point charge}

The formation of cusps in time dependent Hele-Shaw flows have been, and still are, of particular interest, e.g. see [1, 3, 8, 9]. For example, it aids understanding of practical applications such as the extraction of oil which can be modelled by a Hele-Shaw flow driven by a hydrodynamic sink singularity within the oil region [10].

Consider a fluid blob with free boundary, $\partial \Omega(t)$, given by the following polynomial map from the unit $\zeta$-disk:

$$
z=a \zeta+b \zeta^{n}
$$

where $a(t)$ and $b(t)$ are real time varying coefficients, with a point sink located at the origin. For this map it is known that the free boundary evolves through a series of limaçon shapes, eventually leading to cusp formation (see figure 8a) in finite time, $t^{*}$, beyond which the boundary map no longer remains univalent $[3,6]$.

Suppose now that an electric point charge of strength $E<0$ is placed within the fluid domain, with the aim of delaying the formation of the cusp on the boundary, thus enabling a greater proportion of fluid to be withdrawn before the solution breaks down. In $\S 4.2$ it is shown that a negative point charge within the fluid domain provides a symmetrising effect on the boundary about the point charge. A domain with disturbances on its boundary would typically produce cusps over time in sink driven flow. On the other hand the point charge, through its symmetrising effect, would be expected to suppress the development of cusps.

To assess this competition, a fluid blob with initial shape given by $(34)$ with $a(0)=1$, $b(0)=0.1$ and $n=2$, and a point sink of strength $Q=-1$ at $z=0$ is allowed to evolve for the two cases when (a) there is no electric charge at $z=0$ (analytic solution shown), and (b) $E=-5 \pi$. As expected $E=0$ leads to a breakdown of the solution after finite time $t^{*} \approx 1.67$. For $E<0$, the solution lasts far longer $(t>3)$ enabling most of the fluid to be withdrawn (see figure $8 \mathrm{~b}$ ). The boundary can be made to enclose an arbitrarily small region depending on the numerical parameters (time-step, resolution) chosen. Here there 


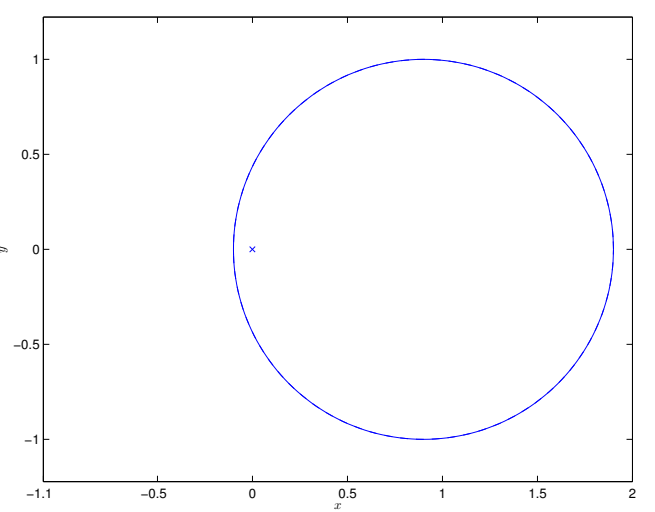

(a) $t=0$

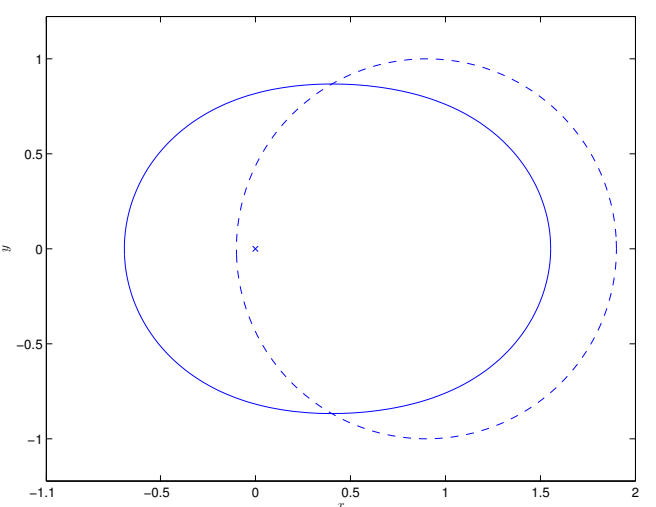

(c) $t=0.85$

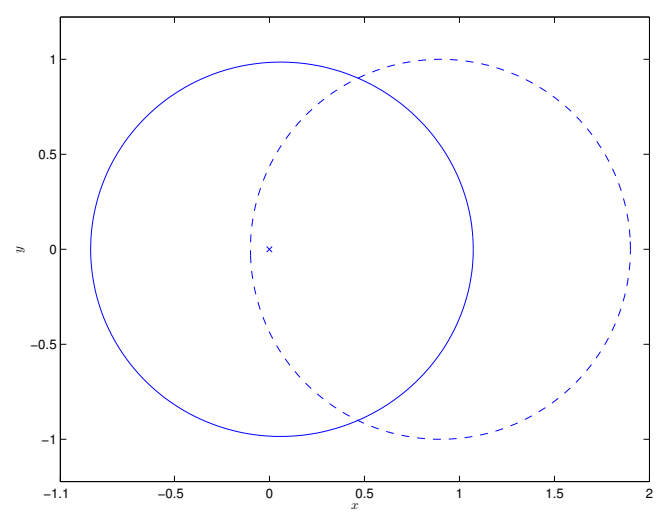

(e) $t=2.85$

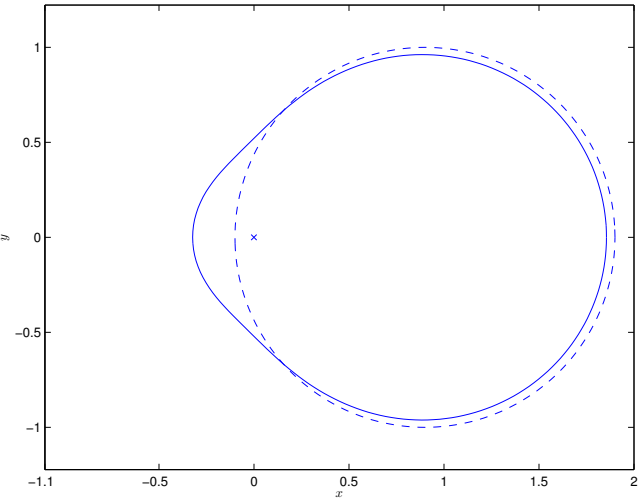

(b) $t=0.1$

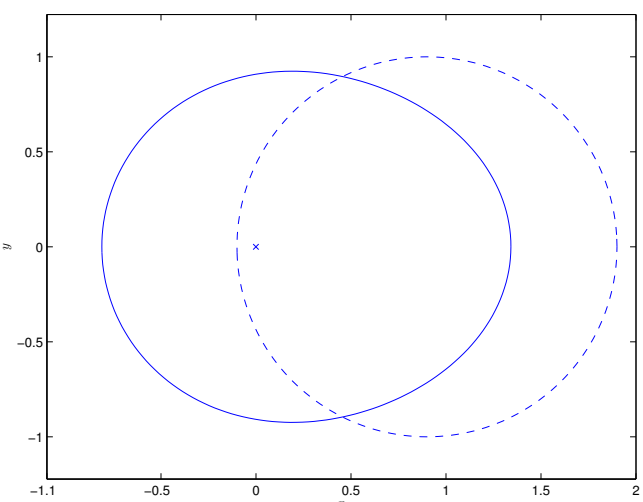

(d) $t=1.45$

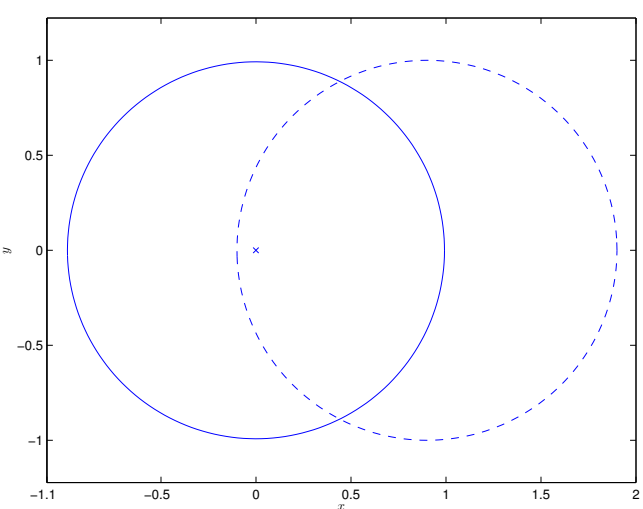

(f) $t=10$

Figure 6: Snapshots showing the evolution of the free boundary (solid) of an initially circular fluid blob (dashed) centred at $\left(\epsilon_{0}, 0\right)$ with $\epsilon_{0}=0.9$ and radius $R_{0}=1$. An electric point charge of strength $E=-2 \pi$ is located at the origin (marked by cross). 


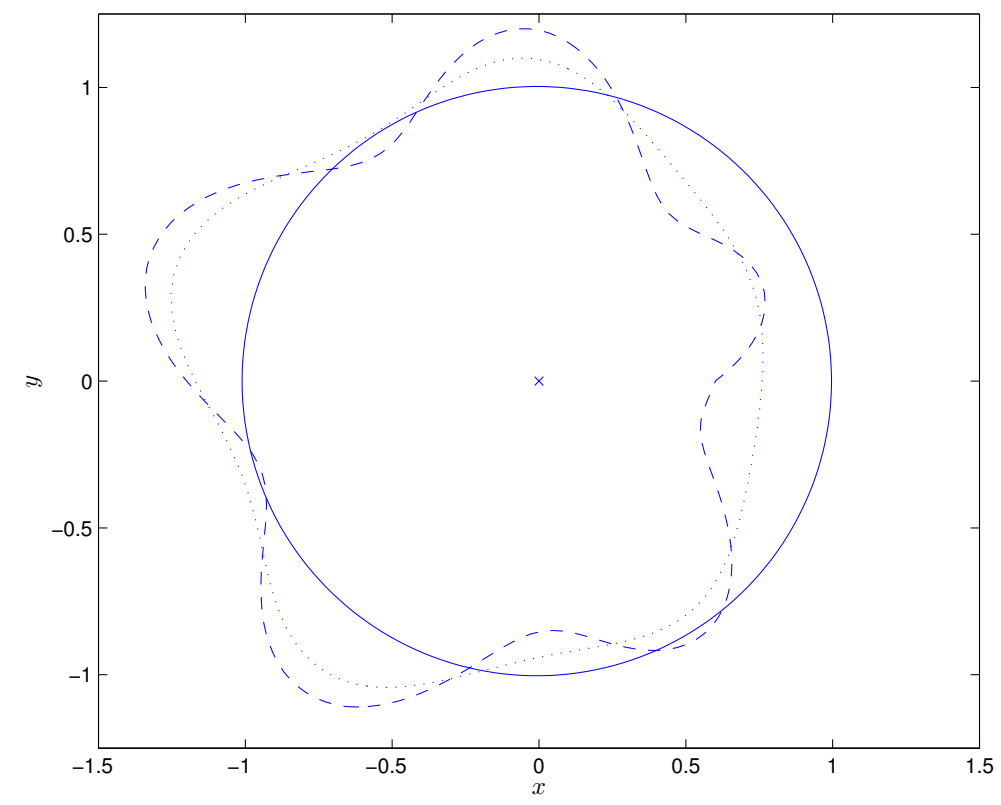

Figure 7: An initial 'random' boundary shape (dashed) with electric point charge of strength $E=-\pi$ located at the origin (marked by cross). Evolution of the boundary is shown at $t=1.2$ (dotted) and $t=22$ (solid). Here there are $N=200$ mesh points on the boundary and step-size $\Delta t=4.89 \times 10^{-4}$.

are $N=100$ mesh points on the boundary and step-size $\Delta t=1 \times 10^{-4}$.

\section{$6 \quad$ Stability of previously found steady solutions}

Explicit steady solutions of the free boundary problem (4) have previously been found by Entov \& Etingof [4] and McDonald [14] involving combinations of hydrodynamic singularities and electric point charges. Here, their stability is tested numerically.

Consider the exact steady solution of Entov \& Etingof [4] given by the boundary map from the unit $\zeta$-disk to the blob in the $z$-plane

$$
z(\zeta)=\sqrt{a b}\left(\frac{1-\alpha \zeta}{1+\alpha \zeta}\right)^{1 / \lambda}
$$

where $\lambda=E / 2 Q$ and

$$
\alpha=\sqrt{\frac{1-(a / b)^{\lambda / 2}}{1+(a / b)^{\lambda / 2}}} .
$$

Here the flow is driven by a point source of strength $Q$ located at $z=a$, a point sink of strength $-Q$ at $z=b(a, b \in \mathbb{R}, b>a)$ and an electric point charge of strength $E$ located at $z=0$, which is outside the fluid domain. The stability of the steady solution is demonstrated numerically for large time (see figure 9a). Simulations are run for $t=T$, where $T$ is a characteristic time scale for the flow calculated as $T \sim L^{2} / Q$, where $L$ is taken 


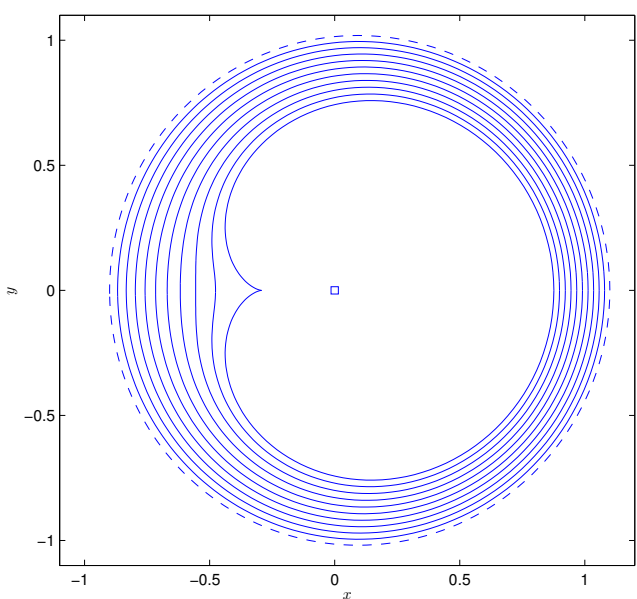

(a)

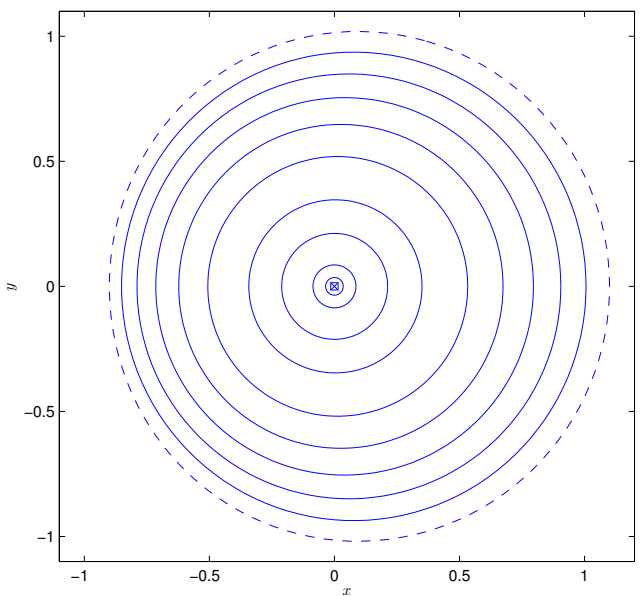

(b)

Figure 8: Comparison between time evolution of free boundaries for (a) cusp formation due to a sink driven flow (analytic solution) with strength $Q=-1$ (marked by square). A cusp forms at $t^{*} \approx 1.67$, the final time shown. (b) Superposition of sink plus electric charge (numerical solution) with $Q=-1$ and $E=-5 \pi$ (marked by square and cross respectively). The final time shown is $t=3.17$. In both (a) and (b) the initial boundary (dashed) is given by the map (34) with $a(0)=1, b(0)=0.1$ and $n=2$.

to be the maximum blob width in the $x$-direction. There is little deviation between initial and final blob shapes after this long time.

The stability of the free boundary subject to an electric point charge lying within the fluid domain is analysed in $\S 2.2$. Since here the electric point charge lies on the exterior of the fluid domain, it is expected that the flow is unstable for negative point charge. Reversing the sign of the source and sink strengths and point charge, the stability of the above steady solution (which is still a steady solution under change of sign of $Q$ and $E$ ) is tested using the timedependent numerical solution. Figure $9 \mathrm{~b}$ shows the rise of instability on the free boundary close to the electric point charge in much shorter time as compared with the characteristic time $T$, and the solution rapidly breaks down. Therefore, the numerical results suggest the solution given by (35) is only stable for positive point charge strength, $E$. The change in stability properties when swapping signs of $E$ and $Q$ is consistent with (5) which implies the two resulting evolutions are time reversals of each other. Figures $9 \mathrm{c}$ and $9 \mathrm{~d}$ show the free boundary at time immediately before breakdown of the solution. The numerical breakdown of the solution could possibly be due to the formation of high curvature or cusp on the free boundary. Alternatively, the breakdown could result from the numerical formulation, as a point charge lying on the free boundary, $\partial \Omega(t)$, would provide a singularity in (32) if a mesh point and the point charge were to coincide.

Figures 10 and 11 show instabilities arising on the boundaries for steady solutions given in $[14]$ by

$$
z(\zeta)=-a \tanh \left(\sqrt{\frac{-\mu}{a E} \zeta}\right),
$$

for a dipole of strength $\mu$ located at $z=0$ and electric point charges $\pm E$ at $z= \pm a(a \in \mathbb{R})$, 


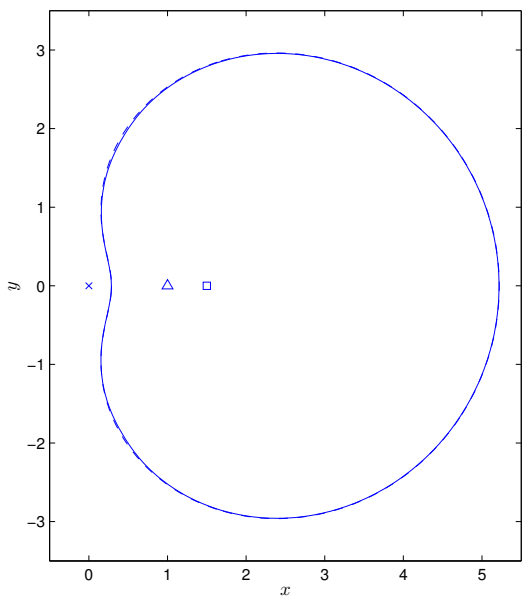

(a) $t_{e}=25, E=0.3909$.

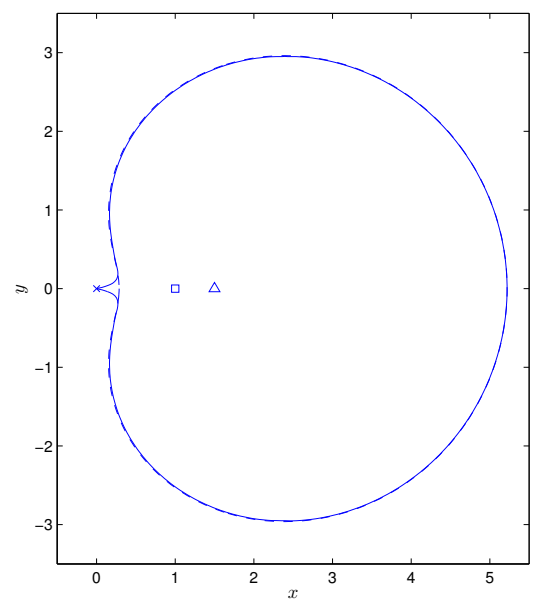

(c) $t_{e}=4.5696, E=-0.3909$.

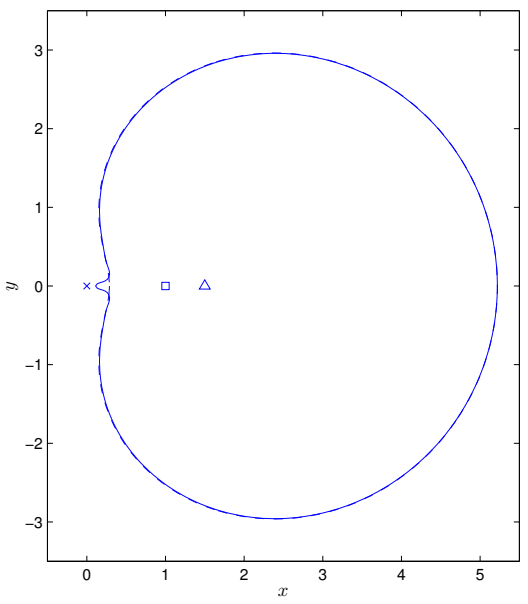

(b) $t_{e}=4.2496, E=-0.3909$.

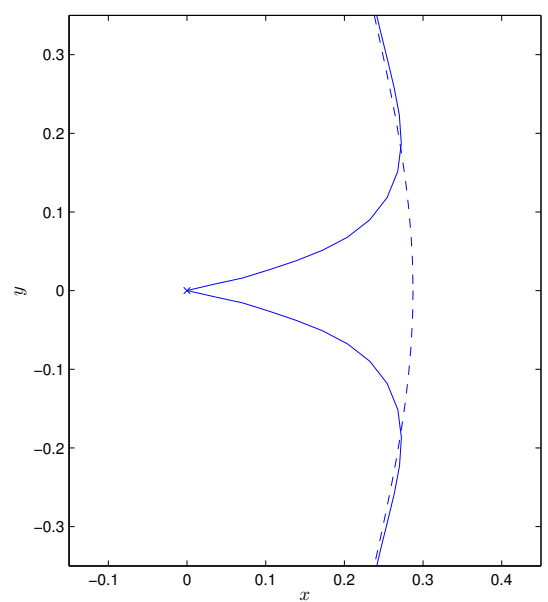

(d) Close up of (c) near point charge, $E=-0.3909$.

Figure 9: Testing stability of the steady solution boundary shape given by (35) for $a=1$ and $b=1.5$, where (a) $E=0.3909, Q=1$. In (b), (c) and (d) $E=-0.3909, Q=-1$. The initial boundary shape (dashed) and at later time $t=t_{e}$ (solid) are shown. The electric point charge, source and sink are marked by a cross, triangle and square, respectively. Here there are $N=500$ mesh points on the boundary, step-size $\Delta t=5 \times 10^{-4}$ and $L=4.9331$ 
and

$$
z(\zeta)=a \sqrt{\frac{1-\exp \left(-\sqrt{\frac{2 \delta}{E}} \frac{\sqrt{a^{2}+b^{2}}}{a b} \zeta^{2}\right)}{1+\frac{a^{2}}{b^{2}} \exp \left(-\sqrt{\frac{2 \delta}{E} \frac{\sqrt{a^{2}+b^{2}}}{a b}} \zeta^{2}\right)}},
$$

with a quadrupole of strength $\delta$ at $z=0$ and electric point charges $-E$ at $z= \pm a$ and $E$ at $z= \pm i b(a, b \in \mathbb{R})$, respectively. Since in both of these exact steady solutions there are negative point charges lying outside of the fluid domain, the flow is unstable. It is evident (within short time) that the instabilities on the free boundary are prominent in regions closest to the negative point charges. Figures 10c and 10d show the free boundary at time immediately before breakdown of the solution when a mesh point lying on the horizontal axis and the point charge coincide. The solution of the free boundary in figure 11 eventually breaks down in a similar manner.

McDonald [14], also gives a family of exact steady solutions for an electric point charge of strength $E$, lying within the fluid domain, super-imposed with a hydrodynamic $n$-pole of strength $M$ at $z=0$, given by

$$
z(\zeta)=\beta \zeta \exp \left(\frac{2 M}{E \beta^{n}} \zeta^{n}\right)
$$

for some parameter $\beta$. The stability of the solution is demonstrated for negative charge $E$ in figure $12 \mathrm{a}$, which shows the case for a hydrodynamic dipole $(n=1)$. The characteristic time scale for the flow is $T \sim L^{3} / M$. The case for positive charge is also tested, and the solution no longer remains steady, developing instabilities on the free boundary and then the solution breaks down rapidly, as expected (see figure 12b). This is due to the aforementioned unstable nature of a positive point charge lying within the fluid blob (c.f. §4.1). Note again that swapping the signs of the forcing and electric charge changes the stability properties.

\section{Discussion}

It has been shown for a Hele-Shaw free boundary flow, if a negative electric point charge lies inside the fluid domain $\Omega\left(t_{0}\right)$, where its boundary $\partial \Omega\left(t_{0}\right)$ is a simple, smooth curve such as a circle, the resulting evolution of the free boundary $\partial \Omega(t)$ is stable. Furthermore, the boundary remains smooth for all time and tends to a steady state which is circular and centred about the point charge. The family of curves through which the boundary evolves depends on the location of the point charge relative to the initial boundary, as displayed in $\S 4.1$. It has been shown analytically, in $\S 2.3$, if the location of the point charge is sufficiently close to the centre of a circular fluid blob, the family of curves the boundary evolves through are approximately circular, for which the numerical results (based on boundary integral methods) show excellent agreement.

It has been shown that for a positive point charge lying within the fluid domain, the evolution of the free boundary is unstable. In $\S 6$, the stability of some exact solutions were tested numerically, where electric point charges of strength $E$ lie outside of the fluid domain. The numerical results suggest for $E>0$, the evolution of the boundary is stable, whilst for $E<0$, it is unstable. 


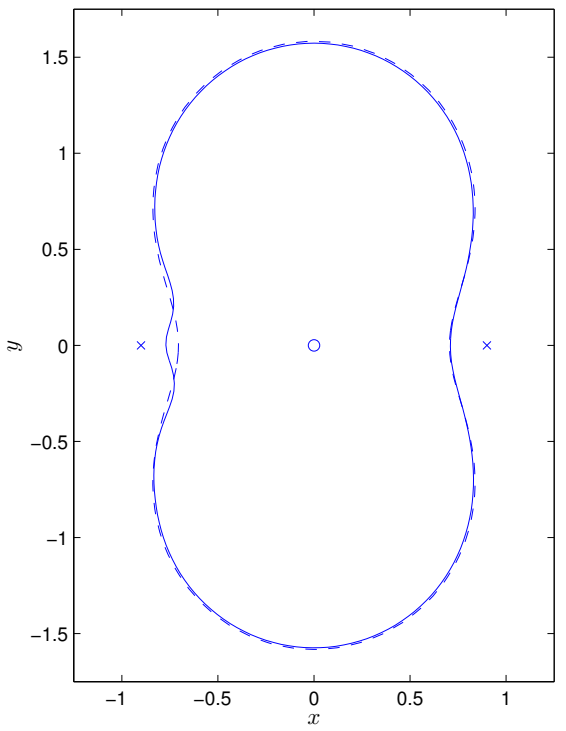

(a) $t_{e}=0.5$

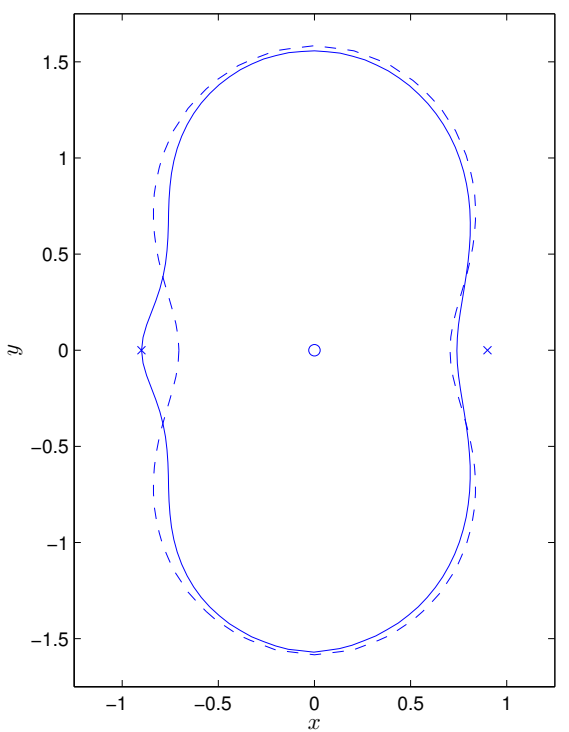

(c) $t_{e}=1.04$

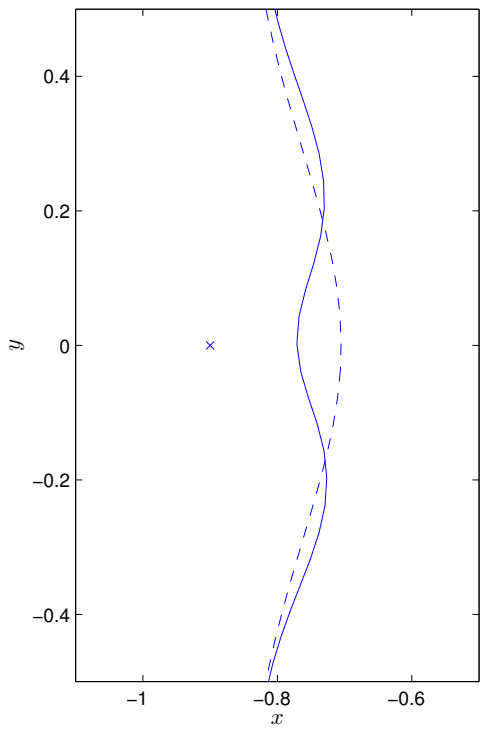

(b) Close up of (a) near negative charge.

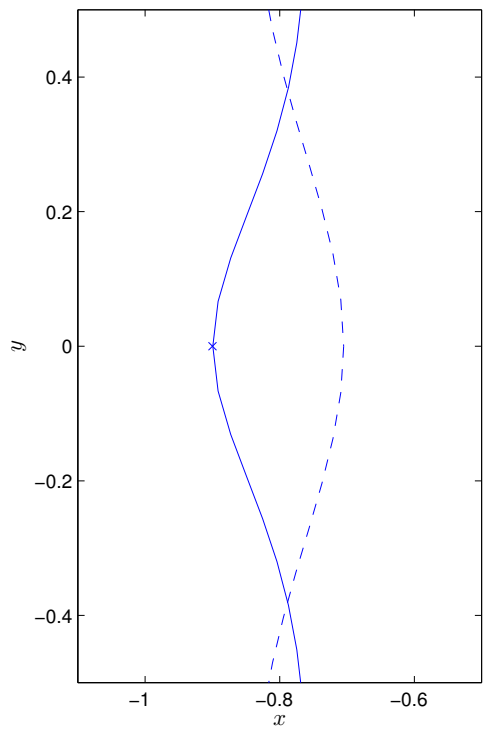

(d) Close up of (c) near negative charge.

Figure 10: Testing stability of the steady solution boundary shape given by (37) for $a=0.9$, $\mu=-1$ with $E= \pm 1$ at $z= \pm a$. Initial boundary shape (dashed) and at time $t=t_{e}$ (solid) are shown. The dipole and electric point charges are marked by a circle and crosses, respectively. Here there are $N=100$ mesh points on the boundary and step-size $\Delta t=$ $1.25 \times 10^{-5}$. 


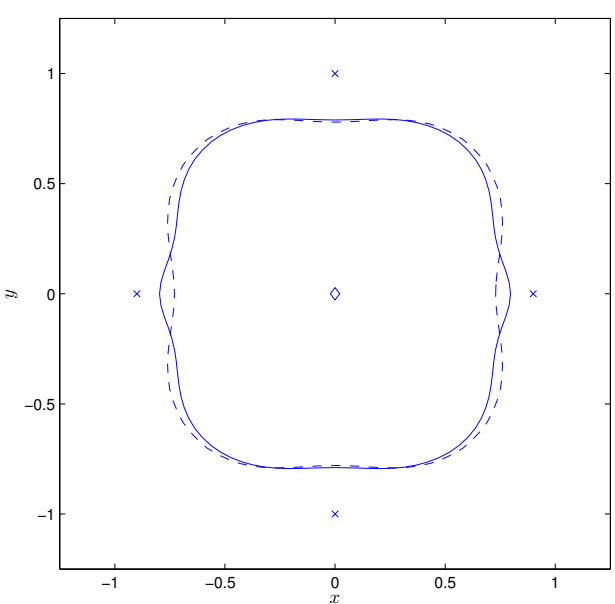

(a) $t_{e}=0.5$

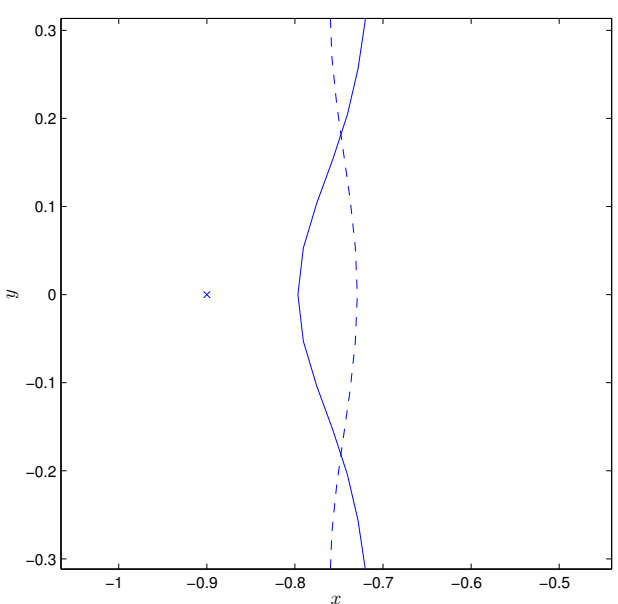

(b) Close up of (a) near negative charge.

Figure 11: Testing stability of the steady solution boundary shape given by (38) for $a=0.9$, $b=1, \delta=0.5$ with $E=-1$ at $z= \pm a$ and $E=1$ at $z= \pm i b$. Initial boundary shape (dashed) and at time $t=t_{e}$ (solid) are shown. The quadrupole and electric point charges are marked by a diamond and crosses, respectively. Here there are $N=100$ mesh points on the boundary and step-size $\Delta t=1.25 \times 10^{-5}$.

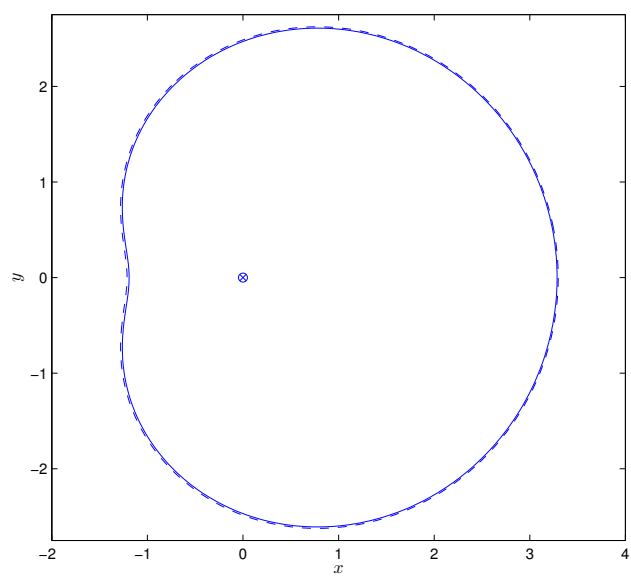

(a) $t_{e}=90, E=-1$.

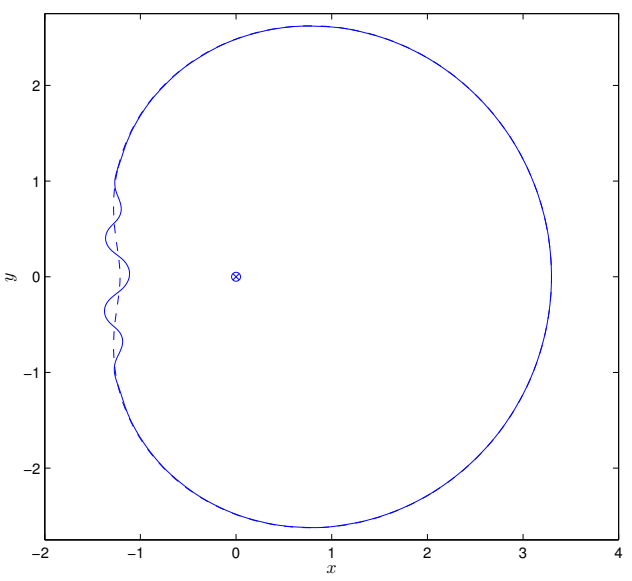

(b) $t_{e}=9, E=1$.

Figure 12: Testing stability of the steady solution boundary shape given by (39) for $\beta=2.0$, $M=E / 2$ and $n=1$, where (a) $E=-1$ and (b) $E=1$. Initial boundary shape (dashed) and at later time $t=t_{e}$ (solid) are shown. The quadrupole and electric point charge are marked by a circle and cross, respectively. Here there are $N=500$ mesh points on the boundary, step-size $\Delta t=3.33 \times 10^{-4}$ and $L=4.4669$. 
For the exact steady solutions given by (35) and (39), numerical results show no instability on the free boundary after some characteristic time scale, provided the sign of electric charge is negative within the fluid blob, and positive when lying outside the fluid blob. Although this does not prove stability or otherwise, by demonstrating that a solution stays close to the original exact, steady solution on some characteristic time scale, then such solutions are likely to be stable. It is important to note the solution breaks down in much shorter time (as compared with the characteristic time) in the corresponding unstable cases.

\section{Acknowledgements}

The first author would like to thank the Department of Mathematics at University College London for providing funding for research through the Departmental Teaching Assistantship programme.

\section{A Numerical filtering}

In many of the computations a 'saw-toothed' appearance of the evolving free boundary, $\partial \Omega(t)$, was observed after a sufficient number of time steps. An example is displayed in figure 13. This phenomenon was also observed in [12], where a similar numerical method is used to model the time evolution of breaking surface waves. As in [12], the source of these 'oscillations' is assumed to arise from the numerical instability of the method employed. In a similar manner to [12], the following five point filtering formula is applied to remedy the problem:

$$
\begin{gathered}
\bar{X}_{i, j}=\frac{1}{16}\left(-X_{i-2, j}+4 X_{i-1, j}+10 X_{i, j}+4 X_{i+1 . j}-X_{i+2 . j}\right), \\
\bar{Y}_{i, j}=\frac{1}{16}\left(-Y_{i-2, j}+4 Y_{i-1, j}+10 Y_{i, j}+4 Y_{i+1, j}-Y_{i+2, j}\right),
\end{gathered}
$$

where $\left(\bar{X}_{i, j}, \bar{Y}_{i, j}\right)$ are the filtered coordinates lying on $\partial \Omega(t)$ at time $t=t_{j}$. These points are then re-noded (via linear interpolation) such that the mesh points are equispaced with respect to arc length, $s$, and denoted by $\left(X_{i, j}, Y_{i, j}\right)$ for $i=1, \ldots, N$, as in $\S 3.2$. Filtering is applied every 10 or 20 time-steps for which the numerical solutions are free from spurious oscillations and in good agreement with existing exact solutions.

As a further test of the effect of filtering on the numerical solutions, consider the polynomial map given in (10), with $n=4$ and $\alpha=0.1$. Simulations are run with and without filtering where the boundary is driven by a hydrodynamic source at an arbitrary location within the fluid domain (see figure 14). Plots in figure 14 verify that filtering has little affect on numerical results (where both filtered and un-filtered solutions are superimposed). However, it should be noted that filtering may have a greater impact on boundary shapes with high curvature, depending on the number of mesh points included. 


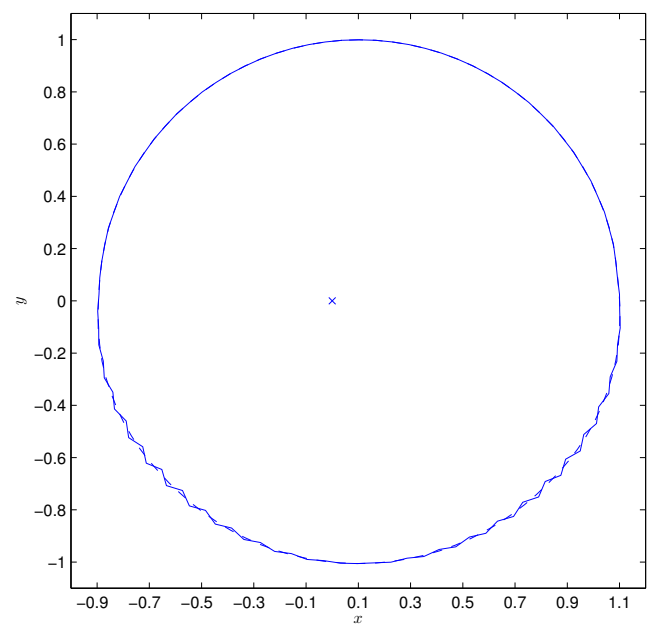

(a)

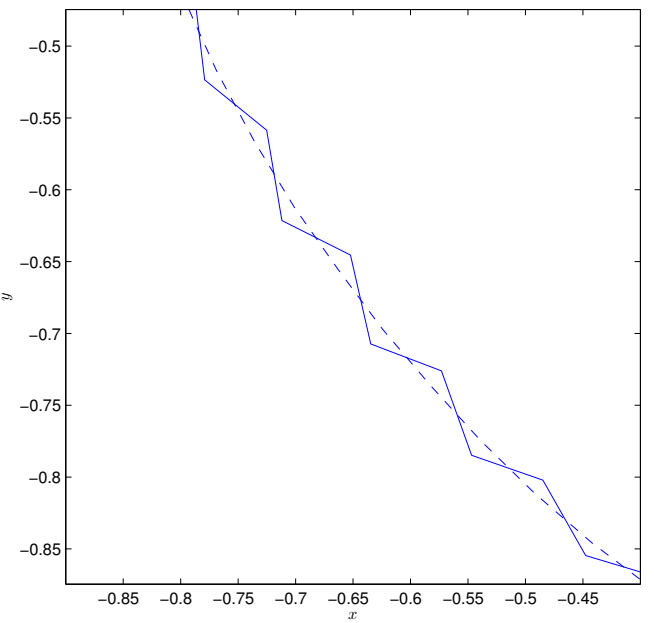

(b)

Figure 13: (a) Snapshot of the evolving free boundary at $t=0.0498$, for an initially circular fluid blob in the case $\epsilon_{0}=0.1, R_{0}=1$, where the fluid blob is subject to an electric point charge of strength $E=-\pi$ located at the origin (marked by a cross). No filtering has been applied. (b) Magnification of boundary displaying 'saw-toothed' effect (solid line) with mesh points alternately lying either side of the filtered smooth boundary (dashed line). Here there are $N=100$ mesh points and step-size $\Delta t=2.5 \times 10^{-4}$.

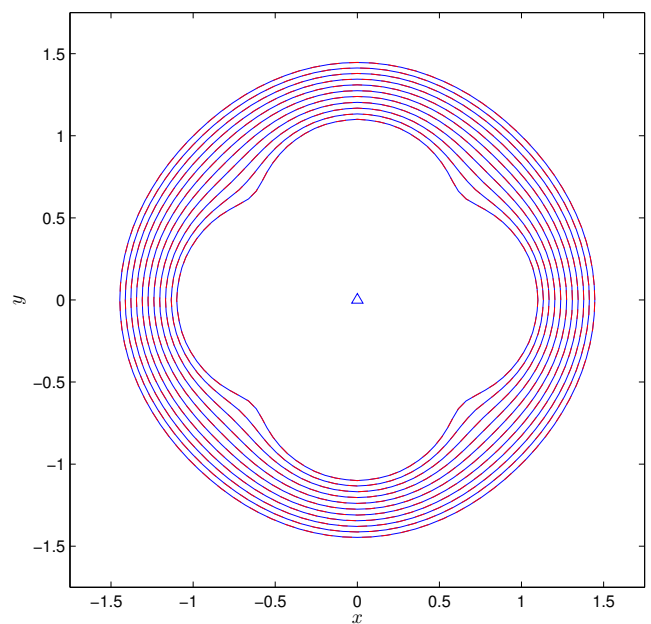

(a) Source at $z=0$

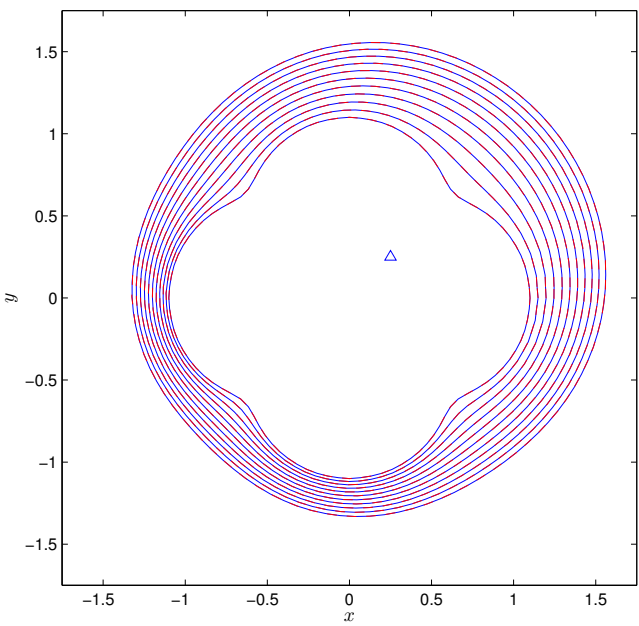

(b) Source at $z=(1+i) / 4$

Figure 14: Superposition of numerical results for filtered (dashed) and un-filtered (solid) data of an initially non-circular fluid blob whose boundary is driven by a hydrodynamic source of strength $Q=\pi$ (marked by triangle), with excellent agreement. Final time shown in both (a) and (b) is $t=1$. Here there are $N=100$ mesh points along the boundary and step-size $\Delta t=1 \times 10^{-3}$. 


\section{References}

[1] Ceniceros, H. D., Hou, T. Y. \& Si, H. (1999) Numerical study of Hele-Shaw flow with suction.Phys. Fluids. 11 (9), 2471-2486.

[2] Cordero, M. L., Burnham, D. R., Baroud, C. N. \& McGloin, D. (2008) Thermocapillary manipulation of droplets using holographic beam shaping: Microfluidic pin ball. Appl. Phys. Lett. 93, 1-3.

[3] Cummings, L. J., Howison, S. D. \& King, J. R. (1999) Two-dimensional Stokes and Hele-Shaw flows with free surfaces. Euro. J. Appl. Math. 10, 635-680.

[4] Entov, V. M. \& Etingof, P. (2007) On generalized two-fluid Hele-Shaw flow. Eur. J. Appl. Math. 18, 103-128.

[5] Entov, V. M., Etingof, P. \& Kleinbock, D. Ya. (1993) Hele-Shaw flows with a free boundary produced by multipoles. Eur. J. Appl. Math. 4, 97-120.

[6] Gustafsson, B. \& Vasilev, A. (2006) Conformal and potential analysis in Hele-Shaw cells. Advances in mathematical fluid mechanics. Birkhäuser Verlag.

[7] Hele-Shaw, H. S. (1898) Flow of water. Nature 59 (1523), 222-223.

[8] Howison, S. D. (1986) Cusp development in Hele-Shaw flow with a free surface. SIAM J. Appl. Math. 46 (1), 20-26.

[9] Howison, S. D., Ockendon, J. R. \& Lacey, A. A. (1985) Singularity development in moving-boundary problems. Q. J. Mech. Appl. Math 38 (3), 333-360.

[10] Kochina, P. Ya. \& Kochina, N. N. (1996) On an oil-field contour. J. Appl. Math. Mechs. 60 (6), 953-957.

[11] Li, D. (2004) Electrokinetics in microfluidics. Interface Science and Technology 2. Elsevier.

[12] Longuet-Higgins, M. S. \& Cokelet, E. D. (1976) The deformation of steep surface waves on water. I. A numerical method of computation. Proc. R. Soc. Lond. A. 350, 1-26.

[13] Malloggi, F., Vanapalli, S. A., Gu, H., van den Ende, D. \& Mugele, F. (2007) Electrowetting-controlled droplet generation in microfluidic flow-focusing device. J. Phys.: Condens. Matter 19 (462101), 1-7.

[14] McDonald, N. R. (2011) Generalised Hele-Shaw flow: A Schwarz function approach. Eur. J. Appl. Math. 1-16.

[15] Mugele, F. \& Baret, J.-C. (2005) Electrowetting: from basics to applications. J. Phys.: Condens. Matter 17, 705-774.

[16] Ockendon, J. R. \& Howison, S. D. (2002) Kochina and Hele-Shaw in modern mathematics, natural science and industry. J. Appl. Math. Mech. 66 (3), 505-512. 
[17] Prakash, M. \& Gershenfeld, N. (2007) Microfluidic bubble logic. Science 315, 832-835.

[18] Richardson, S. (1972) Hele-Shaw flows with a free boundary produced by the injection of fluid into a narrow channel. J. Fluid. Mech. 56 (4), 609-618.

[19] Richardson, S. (1994) Hele-Shaw flows with time-dependent free boundaries in which the fluid occupies a multiply-connected region. Eur. J. Appl. Math. 56 (4), 609-618.

[20] Tristan, U. (2012) MATLAB file available at www.mathworks.com/matlabcentral/ fileexchange/35488-connect-randomly-ordered-2d-points-into-a-minimal-nearest -neighbor-closed-contour.

[21] Vanden-Broeck, J. -M. (1994) Steep solitary waves in water of finite depth with constant vorticity. J. Fluid. Mech. 274, 339-348.

[22] Wong, P. K., WANG, T. H., Deval, J. H. \& Ho, C. M. (2004) Electrokinetics in micro devices for biotechnology applications. IEEE/ASME Trans. Mechanotronics 9 (2), 366-376. 